\title{
Modification at Lattice Scale for an Optimized Optical Response of $\mathrm{Al}_{\mathrm{x}}(\mathrm{ZnO})_{1-\mathrm{x}}$ Nanostructures
}

\author{
Avanish Kumar Srivastava ${ }^{1}$, Karuppanan Senthil ${ }^{2}$, Melepurath Deepa ${ }^{3}$, Ruchi Gakhar ${ }^{1}$, \\ Jai Shankar Tawale ${ }^{1}$ \\ ${ }^{1}$ Materials and Chemical Metrology, National Physical Laboratory, New Delhi, India. \\ ${ }^{2}$ School of Advanced Materials Science \& Engineering, Sungkyunkwan University, Suwon, South Korea. \\ ${ }^{3}$ Department of Chemistry, Indian Institute of Technology, Hyderabad, India.
}

E-mail: aks@nplindia.org

Received February 7, 2011; revised March 14, 2011; accepted March 18, 2011

\begin{abstract}
We report the systematic changes of nano-scaled features and optical properties in a promising transparent oxide material, namely, $\mathrm{Al}_{\mathrm{x}}\left(\mathrm{x}=0,1,2\right.$ and 5\%) doped $\mathrm{ZnO}_{1-\mathrm{x}}(\mathrm{AZO})$. Electron microscopy investigations revealed the alterations at lattice scale depending on the presence of $\mathrm{Al}$-content in $\mathrm{ZnO}$ nanostructures. Lattice spacings of 0.26 and $0.28 \mathrm{~nm}$ oriented along the (0002) and (10 $\overline{10}$ ) planes, respectively, were attributed to euhedral-and facetted-structures of hexagonal-ZnO. The AZO samples were further characterized by XRD, SEM, UV-vis spectrophotometry, Raman spectroscopy and photoluminescence studies. It has been shown that at a dopant concentration of $2 \% \mathrm{Al}$ in $\mathrm{ZnO}$, an optimal balance could be achieved between microstructure and optical properties.
\end{abstract}

Keywords: Electron Microscopy, Nanostructures, ZnO, Photoluminescence

\section{Introduction}

Oxide nanostructures of $\mathrm{ZnO}$ [1-3], $\mathrm{WO}_{3}[4]$ and $\mathrm{TiO}_{2}$ [5] are of great interest due to their tunable microstructure, phase transformation capability and quantum confinement, required for multifunctional usage. Of the lot, $\mathrm{ZnO}$ is an n-type wide band gap semiconductor $\left(E_{\mathrm{g}}\right.$ $3.3 \mathrm{eV}$ ) with a wurtzite hexagonal-crystal structure with excellent chemical and thermal stability, large exciton binding energy $(60 \mathrm{meV})$, large electron mass $\sim 0.3 \mathrm{~m}_{\mathrm{e}}$ $\left(\mathrm{m}_{\mathrm{e}}\right.$ : Bare electron mass) and a large exciton emissivity at room temperature. Doping of $\mathrm{ZnO}$ by transition metal ions such as $\mathrm{Mn}^{2+}[6], \mathrm{Co}^{2+}[7], \mathrm{Ni}^{2+}[8], \mathrm{V}^{3+}[9]$ and $\mathrm{Fe}^{3+}[2]$ yields materials suitable for various optical and electromagnetic applications. Moreover various emission characteristics and shifting of luminescence bands have been attributed to the fine nanocrystalline structures leading to a large surface area and defects [10-12]. Recently neat or undoped $\mathrm{ZnO}$ has also been investigated by incorporating light metal ions like $\mathrm{Al}^{3+}$ for optical applications [13-15]. To this end, in the present study, we report the syntheses and characterization of $\mathrm{Al}$ doped $\mathrm{ZnO}$ nanostructures.

\section{Experimental Details}

Neat $\mathrm{ZnO}$ was prepared by dissolution of 2 gm zinc acetate dehydrate in $25 \mathrm{ml}$ ethanol and $1 \mathrm{ml}$ DEA (diethanolamine), followed by sintering at $500^{\circ} \mathrm{C}$ for two hours. For doped samples, aluminum nitrate nonahydrate was added in terms of weight \% $(1,2$ and 5 wt.\%) and subjected to the same sintering procedure to yield Al-doped $\mathrm{ZnO}$. Hereafter, these samples are referred to as $0 \mathrm{AZO}$, $1 \mathrm{AZO}, 2 \mathrm{AZO}$ and $5 \mathrm{AZO}$ for $0 \% \mathrm{Al}-\mathrm{ZnO}, 1 \% \mathrm{Al}-\mathrm{ZnO}$, $2 \% \mathrm{Al}-\mathrm{ZnO}$ and $5 \% \mathrm{Al}-\mathrm{ZnO}$, respectively. XRD patterns were recorded on a X-ray diffractometer (Mini Flex II) with an incident $\mathrm{Cu}-\mathrm{K} \alpha$ radiation of $\lambda=1.5418 \AA$. A scanning electron microscope (SEM, VP-EVO MA10, Carl Zeiss) and a high resolution transmission electron microscope (HRTEM, JEOL 2200 FS with a Cs-corrector) were employed to characterize the surface and structure of the samples.

\section{Results and Discussion}

A detailed structural and microstructural characterization was carried out on all four samples (0AZO, 1AZO, 


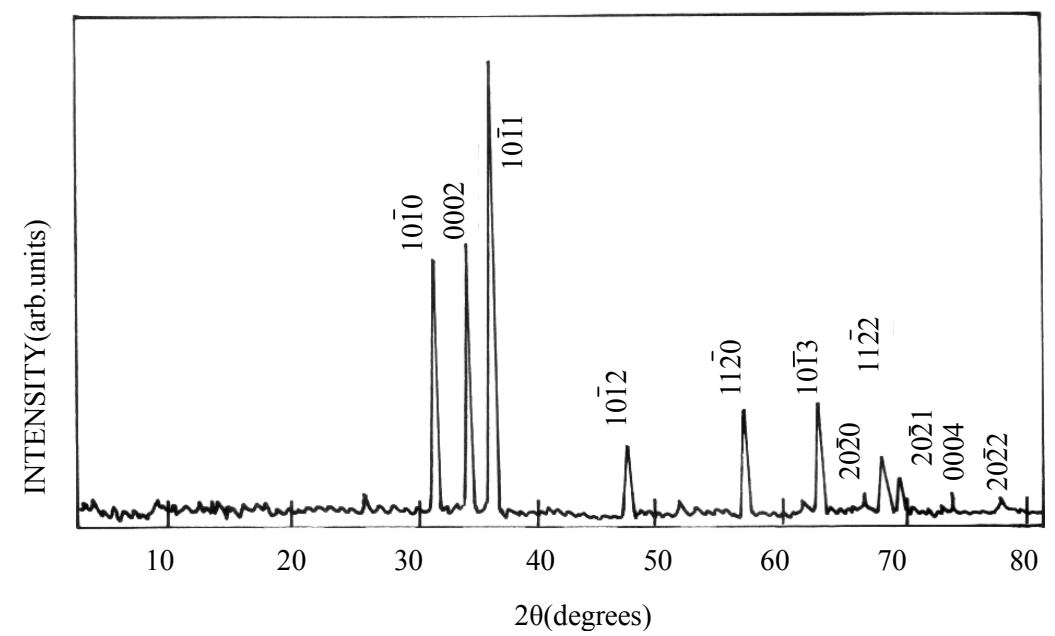

Figure 1. XRD pattern of 0AZO sample showing the presence of important planes of ZnO-hexagonal crystal structure.
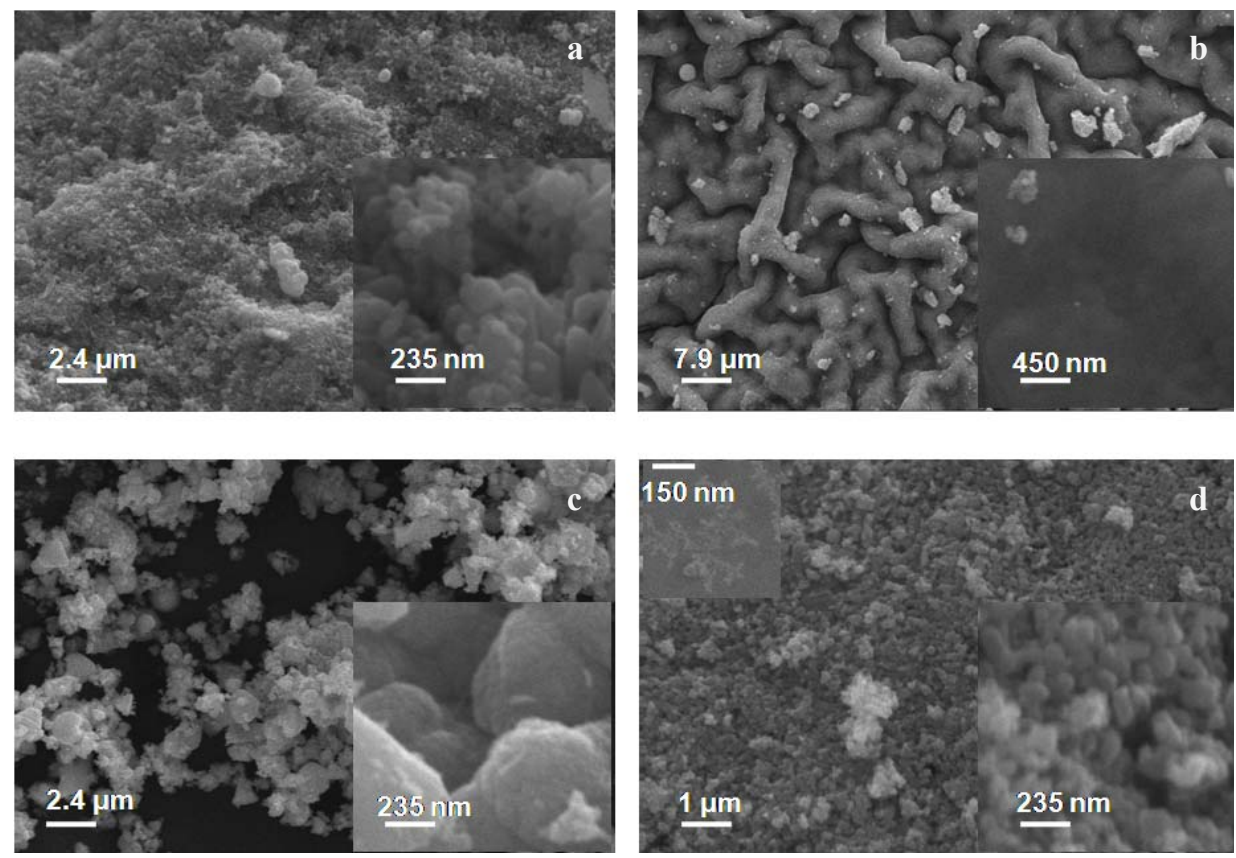

Figure 2. SEM micrographs of AZO samples with $\mathrm{x}=0$ (a),1 (b), 2 (c) and 5 (d) showing the fine topographical features on the surface of these samples. Insets in individual micrographs further reveal the high magnification surface microstructures.

$2 \mathrm{AZO}$ and $5 \mathrm{AZO})$. It was noted that all the four samples exhibited the characteristic peaks of hexagonal $-\mathrm{ZnO}$ (wurtzite type; $\mathrm{a}=0.32, \mathrm{c}=0.52 \mathrm{~nm}$, reference: JCPDS file no. 21-1486) in X-ray diffractogram (XRD). As an illustrative example, a XRD pattern of 0AZO sample exhibiting the intense planes (hkil); $10 \overline{1} 0,0002$, $10 \overline{1} 1,10 \overline{1} 2,11 \overline{2} 0,10 \overline{1} 3,20 \overline{2} 0,11 \overline{2} 2,20 \overline{2} 1,0004$ and $20 \overline{2} 2$ with interplanar spacing (d); 0.28, 0.26, 0.25, $0.19,0.16,0.15,0.14,0.138,0.136,0.130$ and 0.124 , respectively, are marked on diffraction peaks (Figure 1). Further analyses inferred that, initially, the intensity of the $10 \overline{1} 0$ peak decreases in $1 \mathrm{AZO}$, as compared to
0AZO, but ongoing to $2 \mathrm{AZO}$, the intensity of this peak is higher than that of 0AZO. But for the 5AZO sample, the intensity of this peak falls again. The crystallite size estimated from the Scherrer's formula was 5.12, 5.12, 6.14 and $4.72 \mathrm{~nm}$ for $0,1,2$ and 5\% AZO samples, respectively.

The surface topography and microstructures of the samples are analyzed by scanning electron microscopy (Figure 2). These samples are consisted of different morphologies depending on the dopant concentration. In an undoped condition, normally a dense fine size particles are seen (Figure 2a). On increasing the magnifica 

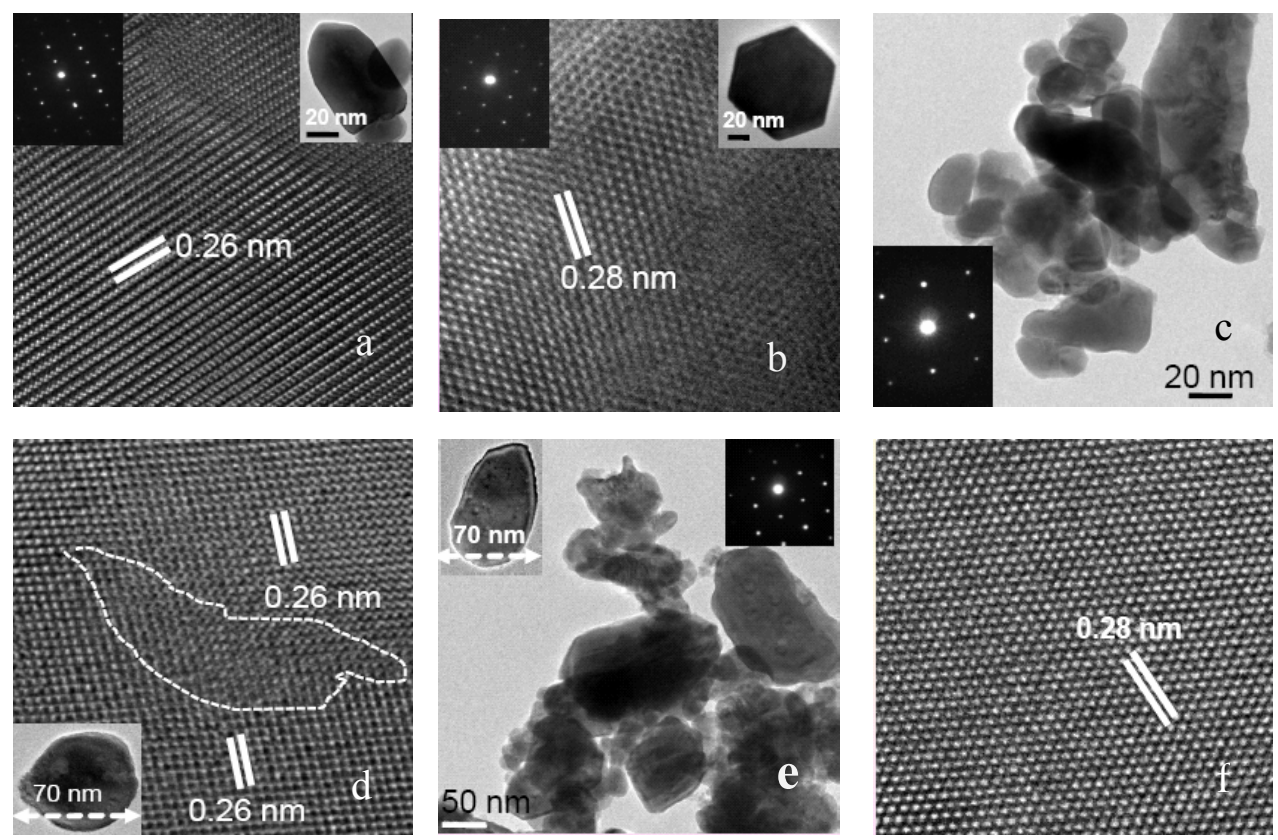

Figure 3. HRTEM micrographs of AZO samples with $x=0$ (a,b), 1 (c,d), 2 (e,f). (a,b) lattice scale images of nanoparticles as shown in insets of (a) and (b). SADPs as shown in the left hand side insets of (a) and (b) correspond to the respective nanoparticles. (c) bright field micrograph and (d) lattice scale image of a nanoparticle (inset of d). Inset in (c) is the corresponding SADP. (e) bright field micrograph, (f) lattice scale image of a nanoparticle shown as an inset in (e). SADP of nanoparticle is also shown as an inset in (e).

tion, the particles with the size between 50 to $150 \mathrm{~nm}$ are resolved in the micrograph (inset of Figure 2a). On addition of $1 \% \mathrm{Al}$ as dopant, there is a change in morphology of the nanoparticles having worm-like appearance with a coarse-structure (Figure 2b) with a very smooth surface (inset of Figure 2b). On further increase in dopant concentration microstructural transformation takes place from worm-like to random particles with carrying more like individual identity (Figure 2c). At high magnification, the surface of these individual particles have developed some cellular microstructure which was seen on every individual nanoparticle (inset of Figure 2c). At highest dopant concentration $(5 \% \mathrm{Al})$ the microstructure is refined (Figure 2d) compared to previous one ( $2 \% \mathrm{Al}$, Figure $2 \mathbf{c})$ and the surface microstructure was also grown (inset of Figure 2d). It is also seen that on increasing the dopant concentration, the agglomeration among nanoparticles increases and it is significant at highest doping (Figure 2d). It is probably due to the fact at high doping, the excess $\mathrm{Al}$, precipitates and goes into the interparticle boundaries and tries to act as binder between the particles. Sometimes the precipitation is so obvious that the excess $\mathrm{Al}$ starts forming its own commonly known dendritic structure (inset as left top corner of Figure 2d).

High resolution transmission electron microscopy was employed to investigate the neat and Al-doped $\mathrm{ZnO}$ samples even at atomic scale and in reciprocal space. In the 0AZO sample, well facetted grains with euhedral shapes (diameter $\sim 80 \mathrm{~nm}$ and length $\sim 180 \mathrm{~nm}$ ) having conical tips were observed (inset of Figure 3a). The corresponding lattice scale image (Figure 3a) shows a set of planes with an interplanar spacing of $0.26 \mathrm{~nm}$ oriented along the 0002 direction. The nanoparticles have a hexagonal structure with each facet of about $40 \mathrm{~nm}$ (inset of Figure 3b). The corresponding lattice scale image (Figure 3b) shows a set of planes with an interplanar spacing of $0.28 \mathrm{~nm}$ oriented along the $10 \overline{1} 0$ reflection. Selected area electron diffraction patterns (SADPs) recorded from a euhedral grain (inset of Figure 3a) oriented along the $[\overline{1} 2 \overline{1} 6]$ zone axis (inset of Figure 3a) and from a hexagonal crystallite (inset in Figure 3b) oriented along the [0001] zone axis (inset of Figure 3b). Since the lattice image in Figure 3b has been recorded along the [0001] zone axis which is also the c-axis of hexagonal $\mathrm{ZnO}$, six planes of oriented along the $\{10 \overline{1} 0\}$ direction are clearly seen. For the 1AZO sample, nanoparticles tend to aggregate and the diameter of these particles varies from 15 to $30 \mathrm{~nm}$ (Figure 3c). A high resolution image recorded from one such nanoparticle (inset of Figure 3d) shows that these particles have lattice scale defects as shown by the encircled region in Figure 3d. On either side of the encircled region, the structure is regular with an interplanar spacing of 0.26 $\mathrm{nm}$ that corresponds to the 0002 plane of hexagonal $\mathrm{ZnO}$. The corresponding SADP, recorded along the [0110] 


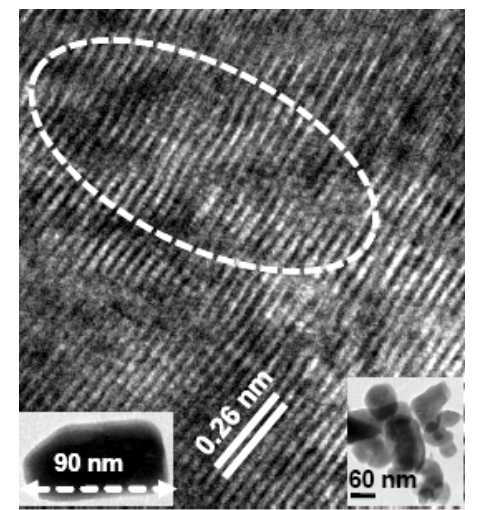

Figure 4. Lattice scale image of a nanoparticle of about $90 \mathrm{~nm}$, shown as inset. The inset on the right hand side shows a cluster of nanoparticles.
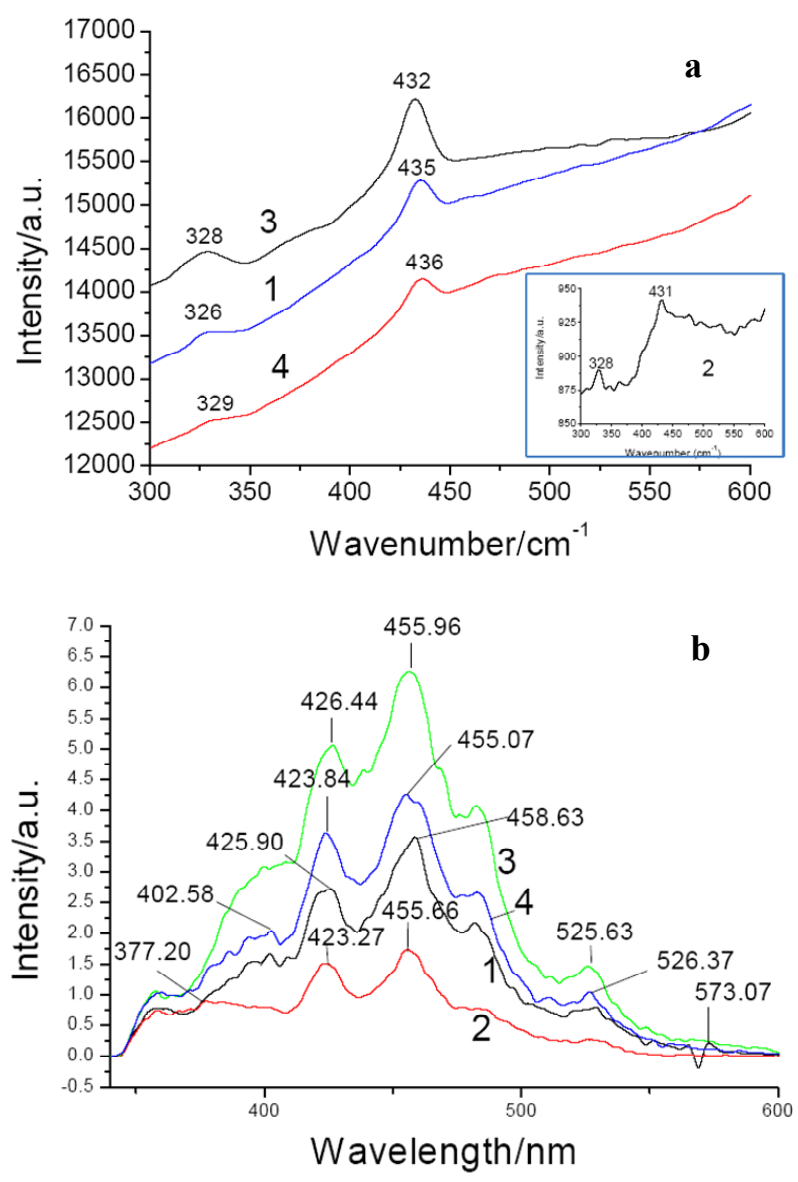

Figure 5. (a) Raman and (b) photoluminescence spectra of AZO samples. $1=0 \mathrm{AZO}, 2=1 \mathrm{AZO}, 3=2 \mathrm{AZO}$ and $4=5 \mathrm{AZO}$.

zone axis is displayed as an inset of Figure 3c. For the 2AZO sample fine nanoparticles (of about $20 \mathrm{~nm}$ diameter) embedded in larger sized particles were seen (Figure 3e). A defect free image recorded along the $\mathrm{c}$-axis of the hexagonal crystal structure (Figure 3f) from a particle of about $70 \mathrm{~nm}$ size (inset of Figure 3e). For the
5AZO sample, dislocations and other prominent defects at lattice scale were observed and these have been encircled by dotted lines (Figure 4). These defects were seen in high resolution image of a nanoparticle of about $90 \mathrm{~nm}$ diameter (inset of Figure 4), isolated from a cluster of nanoparticles (inset of Figure 4). In general the XRD analyses also corroborate the electron microscopy investigations.

Raman spectra recorded at a wavelength of $532 \mathrm{~nm}$ for all AZO samples, shows that the overall features of the spectra remain the same except for the two peaks observed at 328 and $432 \mathrm{~cm}^{-1}$ (Figure 5a). These Raman active modes are ascribed to the multi-phonon process $\left(326 \mathrm{~cm}^{-1}\right)$ and the $\mathrm{E}_{2}^{2}$ high phonon mode $\left(436 \mathrm{~cm}^{-1}\right)$ [13-15]. It is evident from Raman spectra, that the variation in the intensity of the peaks is due to the incorporation of $\mathrm{Al}^{3+}$. Initially for $1 \mathrm{AZO}$ sample, the intensity of the Raman bands decreases as compared to 0AZO but for $2 \mathrm{AZO}$, the intensity increases in comparison to 0AZO (Figure 5a). For 5AZO, the intensity of Raman peaks again decreases. However, the 1AZO sample was characterized by peaks with least intensity (Figure 5a). This behavior may be associated with the change the degree of crystallinity of undoped $\mathrm{ZnO}$ induced by doping with $\mathrm{Al}^{3+}$. From XRD and electron microscopy experiments, the size of the nanocrystallites varies in a same manner as the intensity profile of Raman active bands does. The wurtzite-hexagonal structure of $\mathrm{ZnO}$ is non-centrosymmetric and the $\mathrm{Zn}$ atom $(63.59 \mathrm{amu})$ is about four times heavier than that of oxygen atom $(15.999 \mathrm{amu})$, and therefore the structure is polar. On introducing $\mathrm{Al}^{3+}$ in the $\mathrm{ZnO}$ lattice, the non-polar interaction becomes strong. Initially the structure tries to stabilize by filling the vacancies and defect centers at low dopant levels (1AZO). However in 2AZO, the nonpolar phonon mode $\left(\mathrm{E}_{2}^{2}\right.$ high mode at $\left.436 \mathrm{~cm}^{-1}\right)$ is stronger with respect to that observed for 0AZO. The quality of nanostructured- $\mathrm{ZnO}$ improves with the addition of dopant like $\mathrm{Al}^{3+}$, with $2 \mathrm{AZO}$ being the sample with high crystallinity and a uniform almost defect free structure.

The transmittance spectra recorded within the $300 \leq \lambda$ $\leq 700 \mathrm{~nm}$ wavelength range revealed that the transmittance was almost same $(\sim 50 \%)$ for $0 \mathrm{AZO}$ and $2 \mathrm{AZO}$ samples and this value was reduced for the other two samples, $1 \mathrm{AZO}$ and $5 \mathrm{AZO}$ to about $35 \%$ in the same wavelength range. Photoluminescence spectra of all the four samples of AZO recoded at an excitation wavelength of $320 \mathrm{~nm}$ are shown in Figure 5b. Four bands [2] were seen in all spectra and these have assigned as follows: 1) $377 \mathrm{~nm}(3.29 \mathrm{eV})$ : excitonic emission due to the recombination of excited electron with a hole to form a pair of exciton in the valence band, 2) $425 \mathrm{~nm}$ $(2.91 \mathrm{eV})$ : transition between conduction band $(\mathrm{CB})$ and 
zinc vacancy $\left.\left(\mathrm{V}_{\mathrm{zn}}\right), 3\right) 455 \mathrm{~nm}(2.72 \mathrm{eV})$ : A transition between exciton level (E) and interstitial oxygen (Oi) and 4) $525 \mathrm{~nm}(2.36 \mathrm{eV})$ : a transition between $\mathrm{V}_{\mathrm{o}} \mathrm{Zn}_{\mathrm{i}}$ and valence band. $V_{o}$ represents oxygen vacancies. Among these bands, the emission around $455 \mathrm{~nm}$ (blue) was most intense and the intensity of all the peaks was maximum in case of 2AZO (Figure 5b). On reducing the excitation wavelength to $250 \mathrm{~nm}(4.96 \mathrm{eV})$, the highest intensity peak shifted from $455 \mathrm{~nm}$ to $417 \mathrm{~nm}$. The blue shift from $2.72 \mathrm{eV}$ (blue) to $2.97 \mathrm{eV}$ (violet) when the material was excited with $4.96 \mathrm{eV}$ instead of $3.87 \mathrm{eV}$ radiation, shows that the emission of doped $\mathrm{ZnO}$ nanostructures is tunable, which is beneficial for practical applications of $\mathrm{ZnO}$.

\section{Summary}

The morphology and crystallinity of the AZO samples is dependent on dopant concentration. Raman spectroscopy elucidates the increase in the intensity of $\mathrm{E}_{2}{ }^{2}$ (high) mode, which is non-polar in character and it signifies the reduction in the polar nature of $\mathrm{ZnO}: \mathrm{Al}$ with increase in Al content. A $2 \% \mathrm{Al}-\mathrm{ZnO}$ sample was found to be most suitable for optical applications.

\section{Acknowledgements}

The authors are grateful to the Director, NPL, New Delhi for his permission to publish these results. Mr. K. N. Sood and Dr. N. Bahadur are acknowledged for discussions on ZnO. AKS acknowledges INSA-KOSEF program to carry out research at POSTECH, Korea. Professor K. Yong at POSTECH is gratefully acknowledged.

\section{References}

[1] T. Fukumura, Y. Yamada, H. Toyosaki, T. Hasegawa, H. Koinuma and M. Kawasaki, "Exploration of OxideBased Diluted Magnetic Semiconductors Toward Transparent Spintronics," Applied Surface Science, Vol. 223, No. 1-3, February 2004, pp. 62-67. doi:10.1016/S0169-4332(03)00898-5

[2] A. K. Srivastava, M. Deepa, N. Bahadur and M. S. Goyat, "Influence of Fe Doping on Nanostructures and Photoluminescence of Sol-Gel Derived ZnO," Materials Chemistry and Physics, Vol. 114, No. 10, March 2009, pp. 194-198. doi:10.1016/j.matchemphys.2008.09.005

[3] K. Vanheusden, W. L. Warren, C. H. Seager, D. R. Tallant, J. A. Voigt and B. E. Gnade, "Mechanisms behind Green Photoluminescence in $\mathrm{ZnO}$ Phosphor Powders," Journal of Applied Physics, Vol. 79, No. 1, February 1986, pp. 7983-7990. doi:10.1063/1.362349

[4] M. Deepa, A. K. Srivastava, S. Lauterbach, Govind, S. M. Shivaprasad and K. N. Sood, "Electro-Optical Re- sponse of Tungsten Oxide Thin Film Nanostructures Processed by a Template Assisted Electrodeposition Route," Acta Materialia, Vol. 55, No. 18, October 2007, pp. 6095-6107. doi:10.1016/j.actamat.2007.07.056

[5] A. K. Srivastava, M. Deepa, S. Bhandari and H. Fuess, "Tunable Nanostructures and Crystal Structures in Titanium Oxide Films," Nanoscale Research Letters, Vol. 4, No. 1, November 2008, pp. 54-62. doi:10.1007/s11671-008-9202-9

[6] S. Deka and P. A. Joy, "Synthesis and Magnetic Properties of Mn Doped ZnO Nanowires," Solid State Communications, Vol. 142, No. 4, April 2007, pp.190-194. doi:10.1016/j.ssc.2007.02.017

[7] K. J. Kim and Y. R. Park, "Spectroscopic Ellipsometry Study of Optical Transitions in Zn1-xCoxO Alloys," Applied Physics Letters, Vol. 81, No. 19, August 2002, pp. 1420-1422. doi:10.1063/1.1501765

[8] E. Liu, P. Xiao, J. S. Chen, B. C. Lim and L. Li, "Ni Doped ZnO Thin Films for Diluted Magnetic Semiconductor Materials," Current Applied Physics, No. 3-4, May 2008, pp. 408-411. doi:10.1016/j.cap.2007.10.025

[9] M. E. Koleva, P. A. Atanasov, N. N. Nedialkov, H. Fukuoka and M. Obara, "Role of Vanadium Content in ZnO Thin Films Grown by Pulsed Laser Deposition," Applied Surface Science, Vol. 254, No. 4, December 2007, pp. 1228-1231. doi:10.1016/j.apsusc.2007.07.180

[10] M. Snure and A. Tiwari, "Band-Gap Engineering of Zn1xGaxO Nanopowders: Synthesis, Structural and Optical Characterization," Journal of Applied Physics, Vol. 104, No. 7, October 2008, pp. (073707)1-5.

[11] A. K. Srivastava, M. Deepa, K. N. Sood, E. Erdem and R.-A.Eichel. "Shape Selective Growth of ZnO Nanostructures: Spectral and Electrochemical Response," Advanced Materials Letters, 2011, in press.

[12] J. S. Tawale, K. K. Dey, R. Pasricha, K. N. Sood and A. K. Srivastava, "Synthesis and Characterization of $\mathrm{ZnO}$ Nanostructures for Optical and Antibacterial Applications," Thin Solid Films, Vol. 519, No. 3, November 2010, pp. 1244-1247. doi:10.1016/j.tsf.2010.08.077

[13] D. Behera and B. S. Acharya, "Nano-Star Formation in Al-Doped ZnO Thin Film Deposited by Dip-Dry Method and Its Characterization Using Atomic Force Microscopy, Electron Probe Microscopy, Photoluminescence and Laser Raman Spectroscopy," Journal of Luminescence, Vol. 128, No. 10, October 2008, pp. 1577-1586. doi:10.1016/j.jlumin.2008.03.006

[14] J. U. Brehm, M. Winterer and H. Hahn, "Synthesis and Local Structure of Doped Nanocrystalline Zinc Oxides," Journal of Applied Physics, Vol. 100, No. 6, September 2006, pp. 064311(1-9).

[15] D. Gao, J. Zhang, G. Yang, J. Zhang, Z. Shi, J. Qi, Z. Zhang and D. Xue, "Ferromagnetism in ZnO Nanoparticles Induced by Doping of a Nonmagnetic Element: Al," The Journal of Physical Chemistry C, Vol. 114, No. 32, July 2010, pp 13477-13481. 